\title{
The diagnostic threshold of generalized anxiety disorder in the community: A developmental perspective
}

Katja Beesdo-Baum ${ }^{\text {a, }}$, Susanne Winkel ${ }^{\text {a }}$, Daniel S. Pine ${ }^{\mathrm{b}}$, Jürgen Hoyer ${ }^{\text {a }}$, Michael Höfler ${ }^{\text {a }}$, Roselind Lieb ${ }^{\mathrm{c}, \mathrm{d}}$, Hans-Ulrich Wittchen ${ }^{\mathrm{a}, \mathrm{d}}$

a Institute of Clinical Psychology and Psychotherapy, Technische Universitaet Dresden, Chemnitzer Street 46, 01187 Dresden, Germany

b Section on Development and Affective Neuroscience, National Institute of Mental Health, Bethesda, MD, USA

c Institute of Psychology, Division of Clinical Psychology and Epidemiology, University of Basel, Switzerland d Max Planck Institute of Psychiatry, Munich, Germany

\begin{abstract}
Discussion surrounds the question as to whether criteria for generalized anxiety disorder (GAD) should change, particularly in youth. This study examines the effects of possible criteria changes on GAD prevalence and clinical correlates. DSM-IV GAD was assessed using the M-CIDI in a community sample of adolescents and young adults. Diagnostic thresholds were modified in two age spans (9-20 and 21-34 years) using a person-by-year data file $(\mathrm{N}=38,534$ cases). Relaxing the duration or excessiveness criteria led to the most pronounced changes in GAD prevalence, while relaxing frequency, uncontrollability, or associated-symptom criteria had smaller effects. A lower duration requirement increased rates more in older than younger age spans. Opposite effects occurred for changes in associatedsymptoms or clinicalsignificance criteria. Broader GAD definitions identified cases in both age spans that appeared mostly milder than DSM-IV cases but that still differed from nonGAD cases in various clinical factors and validators. Developmental aspects require stronger consideration in future diagnostic systems.
\end{abstract}

Keywords: DSM-5 Diagnostic criteria Classification Epidemiology Community study

\section{Introduction}

The diagnosis of generalized anxiety disorder (GAD) has been surrounded by controversy. Diagnostic criteria have evolved with each DSM revision, based on efforts to distinguish GAD from normal stress reactions and other disorders as well as to improve reliability (Brown et al., 1994; Brown et al., 2001).

DSM-III (APA, 1980) defined GAD as "generalized, persistent anxiety of at least 1month duration" accompanied by an unspecified number of various other symptoms. In DSM-III-R (APA, 1987) "unrealistic and excessive anxiety and worry (apprehensive expectation) about two ormore life circumstances" was defined as the core feature of the disorder. The required durationwas increased to 6months, and the number of associated symptoms was specified (six out of 18 from motor tension, autonomic and/or vigilance clusters). DSM-IV (APA, 1994) 
made further changes by requiring anxiety and worry (apprehensive expectation) about a number of events or activities to be "excessive" and "difficult to control". Autonomic symptoms were deleted from the criteria leaving six hypervigilance/tension symptoms of which at least three were required for diagnosis (one for youth). As for other disorders in DSM-IV, the GAD diagnosis required clinical significance as demonstrated by impairment or distress.

Recent epidemiological research suggests that the DSM-IV GAD definition may fail to identify a number of patients with clinically significant anxiety and worry. This suggestion is based on analyses varying the threshold for GAD 'caseness' as defined by the DSM diagnostic criteria. In individuals with high levels of anxiety and worry, failure to meet the 6month duration criterion is the most frequent reason for not reaching the DSM-IV diagnostic threshold (Carter et al., 2001; Hoyer et al., 2002; Ruscio et al., 2007). Compared to individuals with six-month duration of symptoms, those with shorter duration are similar in terms of many clinical features such as associated symptoms, age-of-onset, or impairment (Bienvenu et al., 1998; Kessler et al., 2005b; Lee et al., 2009; Wittchen et al., 2002b). This is relevant given findings that unreliability in the determination of the duration criterion largely compromises overall retest-reliability for GAD (Wittchen et al., 1998a).

The 'excessiveness' criterion is controversial due to imprecision in the definition (Ruscio et al., 2005), which also seems to contribute to diminished reliability (Wittchen et al., 1995). However, the excessiveness criterion identifies a unique group of patients in terms of age-ofonset, persistence, and comorbidity (Ruscio et al., 2005). There is little data on the 'uncontrollability' criterion (Andrews et al., 2010). Some research has shown specificity of this criterion in GAD (Hoyer et al., 2002; Hoyer et al., 2001) with some indication for a GAD gradient as evidenced by fairly high rates of uncontrollability in high trait worriers or subthreshold GAD cases (Hoyer et al., 2002; Ruscio, 2002). While retaining the associated hypervigilance/tension symptoms from DSM-III-R in DSM-IV had some empirical support (Marten et al., 1993), the range and required number of associated symptoms had not been evaluated systematically. Recent research suggests that GADis usually associated with more than just three out of the six DSM-IV symptoms (Beesdo, 2006; Brown et al., 1995; Carter et al., 2001).

In preparation for DSM-5, GAD criteria are in need of scrutiny. As part of such an examination, it might be particularly helpful to compare characteristics among individuals meeting the current DSM-IV GAD definition, relative to other definitions, in terms of various clinical factors. Indications for a GAD continuum (e.g. Ruscio et al., 2001) justify the search for different diagnostic thresholds by considering the impact of different criteria on prevalence and key correlates (Ruscio, 2009). Consideration of a range of 'validators' has been promoted (Regier et al., 2009).

It is particularly important to perform such examinations in youth given that DSM-IV applies different definitions of GAD in youth and adults. This difference reflects the fact that DSMIII-R had labeled youth with persistent worries or concerns and associated symptoms as suffering from Overanxious Disorder (OAD). In clinical samples, excellent overlap between DSM-III-R OAD and DSM-IV GAD was found (Kendall and Warman, 1996; Tracey et al., 1997). Moreover, evidence supported the decision in DSM-IV to use lower symptom thresholds for youth relative to adult GAD (Tracey et al., 1997). Nevertheless, epidemiologic data raise questions about applications of DSM-IV GAD definition for youth based on findings on prevalence (lower for GAD than OAD), onset (later for GAD than OAD), and longitudinal course (lack of associations between OAD and later GAD) (Beesdo et al., 2009; 
Beesdo et al., 2010; Bittner et al., 2007; Cohen et al., 1993; Kessler et al., 2005a; Pine et al., 1998; Velez et al., 1989). Specifically, it remains unclear the degree to which changes to the current DSM-IV GAD definition would uniquely affect diagnosis among youth and adults. The current study addresses these issues in a prospective-longitudinal community study among adolescents and young adults.

\section{Methods}

\subsection{Sample}

The prospective-longitudinal Early Developmental Stages of Psychopathology (EDSP) study assessed mental disorders and risk factors in a representative population sample of adolescents and young adults (Lieb et al., 2000; Wittchen et al., 1998c). The sample was randomly drawn from government registries in Munich, Germany. The study emphasized development by sampling 14-15-year-old individuals at twice the probability and 22-24-yearold individuals at half the probability of 16-21-year-old individuals. Sample weights account for this in the subsequent analyses.

$\mathrm{N}=3021$ interviews were conducted at baseline (age range 14-24 years); the response rate (RR) was 70.9\%. At T1 (1.2-2.1 years after baseline), $\mathrm{N}=1228$ interviews were conducted among subjects aged $14-17$ at baseline $(\mathrm{RR}=88.0 \%)$. At $\mathrm{T} 2, \mathrm{~N}=2548$ of the total baseline sample were interviewed $(84.3 \%, 2.8-4.1$ years after baseline); $\mathrm{N}=2210$ at $\mathrm{T} 3(\mathrm{RR}=73.2 \%$; 7.3-10.6 years after baseline). There was no evidence for selective attrition from baseline to 10 -year follow-up for GAD $(\mathrm{OR}=0.9,95 \% \mathrm{CI}$ : $0.5-1.9, \mathrm{p}=.81)$.

The investigation was carried out in accordance with the Declaration of Helsinki. All participants provided written informed consent (for respondents aged 18 years and younger parental consent was provided) after the nature of the procedures had been fully explained. The EDSP program has been approved by the Ethics Committee of the Medical Faculty of the Technische Universitaet Dresden (No: EK-13811).

\subsection{Diagnostic assessment}

Face-to-face interviews were conducted by trained clinical interviewers using the computerassisted Munich-Composite International Diagnostic Interview (DIA-X/M-CIDI) (Wittchen and Pfister, 1997). The DIA-X/M-CIDI allows the assessment of symptoms, syndromes, and diagnoses of 48 mental disorders according to the DSM-IV criteria and the collection of data on onset, duration, severity, and psychosocial impairment. At baseline, the lifetime version of the DIA-X/M-CIDI was used; at each follow-up the interval version.

Test-retest reliability and validity of the DIA-X/M-CIDI diagnoses have been previously reported to range from fair to good (Reed et al., 1998; Wittchen et al., 1998b). The test-retest reliability for GAD was $\mathrm{k}=0.45$ (Wittchen et al., 1998a). Inconsistencies in GAD were mainly due to different responses in regard to the 6-month time duration; retest-reliability of the GAD stem question asking for anxiety/worry of one month or more, however, was good and in the range of other disorders $(\mathrm{k}=0.70)$. Concordant validity of any M-CIDI anxiety diagnosis including GAD compared to independent clinical consensus diagnoses by treating physicians was estimated with $\mathrm{k}=0.79$ (Reed et al., 1998). Age-of-onset reliability is also established with an intra class correlation of 0.97 for GAD (Wittchen et al., 1998a; Wittchen et al., 1999). 


\subsubsection{Assessment of $G A D$}

The M-CIDI/GAD section starts with a screening question: "Now I want to ask you about longer periods of feeling worried, tense or anxious. Have you ever had a period of a month or more when most of the time you felt worried, tense, or anxious about everyday problems?" The entire section was skipped if the respondent answered no; else the respondents were asked to report the longest period that they felt worried followed by questions assessing all DSM-IV criteria for GAD (at baseline among those with at least three months of worrying only). Onset was assessed at the end of the section by asking when the first time was that they were anxious and worried over several months. Recency was assessed by asking "When was the last time you felt anxious, tense or worried often or most of the time for a period of 4 weeks or more?" For respondents reporting that onset/recency occurred "within the past 2 weeks" to "within the past 6-12 months", the age at interview was used; respondents reporting onset/recency "more than 12 months ago" were asked how old they were then.

For the present analysis, the exclusion criteria for GAD were not applied, following procedures in prior studies (Beesdo et al., 2010; Carter et al., 2001; Ruscio et al., 2007). To examine effects of each criterion change, each specific criterion was systematically varied to create unique definitions. Cases meeting criteria for these alternative definitions then were compared to cases meeting DSM-IV criteria, while applying the same GAD criteria at all ages, including 3 associated symptoms, and to non-cases (no DSM-IV or subthreshold GAD). Specifically, the duration (criterion A-1) was relaxed from six to three months (1- or 2-month GAD was not considered because it was not assessed at baseline). Associated symptoms (criterion C) were eased from at least three to at least one symptom (following the DSM-IV rule for GAD in children). Worrying more days than not (criterion A-2), excessiveness (criterion A-3), uncontrollable worries (criterion B), and clinical significance (criterion E) were relaxed by skipping them.

\subsubsection{Assessment of other mental disorders}

Following DSM-IV/M-CIDI diagnoses were used to analyze comorbidity among subjects with different GAD definitions: Any other anxiety disorder (panic disorder, agoraphobia, social phobia, specific phobia, obsessive-compulsive disorder), any depressive disorder (major depression, dysthymia), and any substance use disorder (alcohol or illicit drug abuse/dependence).

\subsection{Other measures}

Help-seeking was assessed at the end of each DIA-X/M-CIDI interview by asking respondents if they have ever (baseline) or since the last interview (follow-up assessments) contacted a medical doctor or another health professional due to psychological problems. Persistence of GAD was defined using age-of-onset and age-of-recency information of the respective assessment for all cases that reported at least some GAD symptoms. The score ranges between 0 and 1 and reflects the proportion of years spent with symptoms since first onset of the condition.

Based on prior findings (Beesdo et al., 2010; Goldberg, 2008; Hettema, 2008; Kessler et al., 2008; Moffitt et al., 2007) the following measures were used as "validators" for GAD: 
Parental psychopathology. As previously reported (Beesdo et al., 2010; Lieb et al., 2002), parental diagnoses were based on two sources: (1) direct information from parent interviews (DIA-X/MCIDI) at T1/T3 and (2) family history information as assessed from the offspring at T0/T2/T3 using a modified version of the Family-History- Research-Diagnostic-Criteria (Merikangas et al., 1998) as a model. Parental disorders were aggregated using a priority hierarchy: First, direct information from T1/T3 was used if available, followed by T3 family history reports, then $\mathrm{T} 2$ family history reports, and last $\mathrm{T} 0$ family history reports. In the current paper, we consider the following parental disorders: GAD (irrespective of comorbidity), other anxiety disorders (specific phobia, social phobia, agoraphobia, panic disorder), depressive disorders (major depression, dysthymia) and substance-use disorders (alcohol and illicit drug abuse/dependence), whereby the latter three groups exclude cases with GAD.

Temperament/personality. The German version of the Retrospective Self-Report of Inhibition (RSRI, Reznick et al., 1992) was used at baseline to assess "Behavioral Inhibition (BI)", defined as consistent restraint in response to both social and nonsocial situations. The psychometric properties of the German RSRI were found to be comparable to those of the English version (Rohrbacher et al., 2008).

The German Version of the 100-item Tridimensional Personality Questionnaire (TPQ, Weyers et al., 1995) was used at T3 to assess 11 subscales, loading on three distinct dimensions: novelty seeking, reward dependence and harm avoidance (Cloninger, 1987a; b). Reliability and construct validity of the German Version indicate sufficient psychometric properties (Weyers et al., 1995). Environmental factors. Early adversity was reflected by separation events and/or trauma before the age of 10. Early separation events were assessed at baseline within the family history section of the DIA-X/M-CIDI and included death of parent and separation/divorce of parents. Exposure to trauma was assessed within the DIA-X/MCIDI section on posttraumatic stress disorder at baseline using a list of 10 specified events (e.g. physical threat/violence, rape, serious accident) and one open question on any other traumatic event. Consistent with the DSM-IV A2 criterion, only "qualified" traumas were considered, i.e. respondents reported intense fear, helplessness, or horror when experiencing the traumatic event.

Ninety-one different life events and conditions in 11 role areas including e.g. school/education, family, social contacts, and living circumstances, were assessed with the Munich Event List (MEL, Maier-Diewald et al., 1983). At baseline, the years 1990-1995 were covered, at T2 1995-1998, and at T3 1998-2004. The weighted total life event scores were used herein. Individual items were first weighted according to expert ratings and then combined to form an index (Friis et al., 2002).

\subsection{Statistical analyses}

Accounting for the 'waxing and waning' nature of disorder symptomatology over time (Wittchen et al., 2000), a person-byyear- of-age data file was created. This file indicated the years of age during which a disorder was 'active', considering the various thresholds, and this filewas used to conduct analyses in specific age spans (youth vs. young adulthood). The person-by-year-of-age data file contained one row in the data set for each age-year of life of a subject, spanning the 5 years before baseline until the age at the last completed assessment. Only information up to 5 years before baseline assessment was included to reduce bias in recall of past history. With this approach, each person generated multiple "cases". For instance, a participant aged 17 at $\mathrm{T} 0$ and 27 at last assessment contributed $16(5+11)$ person- 
by-year rows, from ages 12 through 27, to the data set, representing his respective GAD status (depending on which criteria were used) at each of these 16 ages. Overall, data from 3021 participants were transformed into a data set with a total of 38,534 person-by-year-of-age data rows, with variables being correlated within individuals (see below). Note that a total of 9007 interviews were completed at the four assessments $(3021+1228+2548+2210)$. With $\mathrm{Nc}=$ 38,534 person-by-year cases this means that on average 4.3 retrospective years of age are covered by one assessment. Age here ranged from 9 to 34 years.

Age-of-onset and age-of-recency information were used to code which person met the respective applied GAD criteria during which year of age; that is, the interval defined by ageof-onset and age-of-recency in that person had to include the respective year. In the example above, if, in a particular person, the onset age for a definition of GAD was 15 and the recency age was 17, a "zero" would be coded in each data row for age years 12 through 14 . A "one" would then be coded at ages 15 through 17, and a "zero" again at ages 18 through 27 . Thus, this person counts 3 times as GAD case of a particular definition and 13 times as non-case. This is the data pattern that one would (in the absence of recall bias) expect when conducting 16 subsequent cross-sectional studies each addressing the presence/absence of the disorder in the respective year of age. When putting the information from different assessment waves together in this manner, the information for the interval between prior assessment and respective assessment was used. In the example, T0 information was used to cover the ages 12 through 17, T1 information was used for ages 18 and 19 (if the person was 19 years at T1), T2 information was used for ages 20-22 (if the person was 22 years at T2), and T3 information was used for ages 23 through 27. In this way, persons reporting GAD at more than one assessment were coded according to the respectively reported GAD status at the respective ages. Thus, in contrast to a more conventional person-level data file, our person-by-year of age data file considered the potential heterogeneity in GAD expression within persons over time.

Analyses were then conducted in the total sample (9-34 years) and e referring to substantive questions as proposed by the DSM-IV (different GAD criteria in youth vs. adults) e in two different age spans that reflect developmental phases for late childhood/adolescence (9-20 years; unweighted $\mathrm{Nc}=22426$, weighted $\mathrm{Nc}=18785)$ and early adulthood $(21-34$ years, unweighted $\mathrm{Nc}=16108$, weighted $\mathrm{Nc}=19836)$. We did not adjust the significance level $(\mathrm{p}<$ .05 ) for multiple testing since a) our aim in this work is to provide explorative analyses creating hypotheses and stimulating further research, b) different age spans were used following assumptions in DSM-IV, and c) we did not want to penalize the analyses in one age span by analyzing another age span.

Data and results ( $\%$, ratios, statistical inference) were weighted by sex, age and geographic location at baseline to match the distribution of the sampling frame on the level of the person (Lieb et al., 2000) and then applied to all person-by-year of age cases per person; number of cases (Nc, nc) are reported unweighted. Scores (behavioral inhibition, personality scales, life event index) were standardized $(\mathrm{M}=0 ; \mathrm{SD}=1)$. The Stata Software package 10.1 (Stata Corp, 2009) was used for all calculations. Confidence intervals (95\% CI) and p-values were calculated robustly against both weighting and correlations between person-by-year of age cases within persons. The corresponding sandwich estimator of variance in regressions assumes that observations are independent across respondents but allows for dependencies within respondents (Royall, 1986). This ensures valid statistical inference for multiple dependent cases per person. Associations in terms of Odds Ratios (OR) were revealed from logistic regression analyses. Statistical significance was evaluated at the $\mathrm{p}<.05$ level. 


\section{Results}

\subsection{The prevalence of GAD per person-year as a function of threshold level and age}

The first analysis used adult DSM-IV GAD criteria in all individuals, regardless of current age (that ranged from 9-34). Thus, the definition required at least three out of six associated symptoms even in the young, a stricter definition than in DSM-IV. In this analysis, the prevalence of DSM-IV GAD in the total person-by-year data set (which generates data comparable to 12-month prevalence rates derived from cross-sectional epidemiological investigations) was $0.60 \%(\mathrm{nc}=190)$. The prevalence was significantly higher in the older age span $(0.76 \%, \mathrm{nc}=120$ from ages $21-34)$ relative to the younger age span $(0.44 \%, \mathrm{nc}=70$ from ages 9-20; OR $=1.7,95 \% \mathrm{CI}: 1.2-2.4)$ and higher in females $(0.79 \%, \mathrm{nc}=122)$ than in males $(0.42 \%, \mathrm{nc}=68$; OR $=1.9,95 \% \mathrm{CI}: 1.3-2.6)$.

Many potential GAD cases $(1.0 \%, \mathrm{nc}=375$ of the total data-by-year data set) fell below the DSM-IV-threshold by one criterion; a further $1.0 \%(\mathrm{nc}=299), 0.32 \%(\mathrm{nc}=106)$, and $0.23 \%$ $(\mathrm{nc}=76)$ by two, three, and four criteria respectively. Focusing on the subgroup of cases that were just one criterion short ( $\mathrm{nc}=375$, Fig. 1$), 60.2 \%$ were not diagnosed due to duration and $27.0 \%$ did not endorse excessiveness. Interestingly, older cases were more likely than younger cases to fall below DSM-IV-threshold due to insufficient duration. Vice versa, younger cases were more likely than older cases to fall below diagnostic threshold because they endorsed fewer than three associated symptoms or because they failed to report clinical significance criteria.

Consistent with these observations, broadening the DSM-IV GAD definition resulted in varying prevalence increases in the total person-by-year file $(\mathrm{Nc}=38,534)$ based on number and type of diagnostic criteria relaxed (Table 1). Focusing on one relaxed criterion, GAD rates in the total sample increased by $60.0 \%$ when duration was required to be only three months and by $46.7 \%$ when anxiety was not required to be excessive. The rate of GAD more than doubled when both these criteria were relaxed simultaneously. Considerably smaller prevalence increases occurred for other definitions varying one or two criteria. ${ }^{1}$

Importantly, the varying prevalence increases were partly age-dependent. Greater percentage changes were found in the older age span when broadening the duration criterion. Vice versa, smaller percentage changes in higher age occurred for the associated symptom and clinical significance criteria. Irrespective of which GAD criterion was relaxed, however, significantly higher GAD rates were found consistently in the older age span compared to the younger age span.

In the DSM, each diagnostic criterion is assumed to contribute significantly toward diagnosis. The frequency, uncontrollability, and symptom criteria were rarely the reason for nondiagnosis if individuals were short just one criterion. This raises questions on the degree to which these criteria are 'necessary' or are redundant with other criteria. Table 2 shows the associations between all of the GAD criteria among all cases reporting at least three months of worrying (no further criteria necessarily required; $\mathrm{nc}=739$ ). In the total sample, highest associations were found between the uncontrollability and the excessiveness criterion ( $\mathrm{OR}=$ 5.3). Thus, one could ask whether a worry can be excessive but still controllable; this was rare in our data set $(8.9 \%$ of those with three month long excessive worry, $4.4 \%$ of those meeting

\footnotetext{
${ }^{1}$ Note: For subthreshold GAD with 2 relaxed criteria, combinations refer only to criteria with larger case numbers omitting "A-2: worrying more days than not" and "B: uncontrollability".
} 
all other GAD criteria). Some more cases (37.7\% of those with 3 month long worry; $31.5 \%$ of those meeting all other GAD criteria) rated their worry as uncontrollable but not excessive. Analyses by age revealed that the excessiveness-uncontrollability association was found in both age spans but was particularly pronounced in the young. In the older age span, frequency and uncontrollability showed the strongest association.

\subsection{Clinical factors and validators}

Clinical factors and validators are first examined for DSM-IV GAD relative to all other individuals (subthreshold and non-cases), followed by comparisons of subthreshold cases to DSM-IV cases and to non-cases. Because relaxing the frequency and/or uncontrollability criteria identified few cases (compare Fig. 1 and Table 1), due to power, the subsequent analyses consider only subthreshold definitions with one or two of the following criteria relaxed: duration, excessiveness, associated symptoms and clinical significance. To explore developmental effects, analyses in the total sample are followed by results in the two age spans (Tables available upon request).

\subsubsection{Comorbidity}

DSM-IV GAD was associated with comorbidity. $90.7 \%$ of cases that met full GAD criteria (same criteria applied at all ages) manifested comorbidity with at least one other DSM-IV disorder, compared to only $23.6 \%$ of all other individuals, reflecting high same-year associations $(\mathrm{OR}=31.0,95 \% \mathrm{CI}$ : 18.8-51.2). Other anxiety disorders were the most frequently observed comorbid condition $(58.0 \%$ vs. $11.5 \%, \mathrm{OR}=10.3,95 \% \mathrm{CI}$ : $7.4-14.5)$ followed by depressive disorders $(45.4 \%$ vs. $6.0 \%, \mathrm{OR}=11.7,95 \% \mathrm{CI}: 8.3-16.5)$ and then substance use disorders (30.1\% vs. $10.4 \%$, OR $=5.0,95 \% \mathrm{CI}: 3.6-7.1)$. Associations were also significant in both age spans.

Compared to cases meeting full DSM-IV GAD criteria, comorbidity with 'any' disorder was significantly lower in cases identified by relaxing only one or two criteria (see OR1 in Table $3)$. For the specific anxiety, depressive, and substance use disorder groups, however, significance was more consistently indicated only when relaxing at least two criteria.

Although for many subthreshold GAD definitions, cases thereby identified manifested lower comorbidity than full-criteria DSM-IV GAD cases, anxiety, depressive and substance use disorders typically still were occurring more frequently in those subthreshold cases compared to non-GAD cases (see OR2 in Table 3). Performing the same analysis in the two different age spans generally revealed similar findings (results available upon request).

\subsubsection{Help-seeking}

Cases that met full DSM-IV GAD criteria sought help more often $(64.4 \%$; OR $=11.9,95 \%$ CI: 8.3-17.1) compared to those with either subthreshold or no GAD (12.4\%). Relative to DSM-IV GAD cases, helpseeking rates were not significantly lower in subthreshold cases with only one less criterion. When relaxing two criteria, however, some significant reductions were found (duration $\geq 3$ months and excessiveness: $\mathrm{OR}=0.6,95 \% \mathrm{CI}: 0.4-0.9$; excessiveness and clinical significance: $\mathrm{OR}=0.5,95 \% \mathrm{CI}$ : 0.3-0.9). Yet, compared to cases without subthreshold or DSM-IV GAD, cases with subthreshold GAD alone still showed -despite to a lesser extent- significant associations with help-seeking. These associations occurred similarly in both age spans with the exception that older cases without clinical significance did not reveal a significantly higher help-seeking rate than non-cases. This contrasts with young cases 
without clinical significance that did seek help significantly more often than non-cases $(\mathrm{OR}=$ 7.8, CI: 1.7-36.2) (and similarly often as DSM-IV cases: OR =0.8, CI: 0.2-4.3).

\subsubsection{Persistence}

Cases that met full diagnostic criteria for DSM-IV GAD are characterized by persistent GAD symptoms. For such cases, in the total sample, more than half of the time observed since onset of the condition was spent with symptoms (percent time: $\mathrm{M}=0.53, \mathrm{SD}=0.31$ ). When the same criteria were applied at all ages requiring at least three associated physical symptoms also in the young, persistence tended to be similar in both ages $(9-20$ years: $\mathrm{M}=0.52, \mathrm{SD}=$ 0.26 ; $21-34$ years: $\mathrm{M}=0.54, \mathrm{SD}=0.33$ ).

In the total sample, subthreshold GAD cases identified by one or two relaxed criteria showed significantly lower persistence (Range M: 0.26-0.48) compared to DSM-IV cases in all instances. A noteworthy exception was the associated symptom criterion where, persistence significantly increased when the GAD definition was relaxed by one criterion $(\mathrm{M}=0.72)$. Interestingly, this increase in persistence was due to high persistence in the older age span $(M=0.95)$, while in the younger age span persistence was similar to that for cases with DSMIV GAD $(\mathrm{M}=0.48)$. Other subthreshold definitions generally resulted in lower persistence compared to DSM-IV GAD in both age spans.

\subsubsection{Predictors/validators}

Similar to a previous report (Beesdo et al., 2010), full DSM-IV GAD was associated with parental disorders, with temperament/ personality characteristics, and environmental risks (Table 4). Focusing on age span differences in associations, some notable findings emerged e as also indicated by significant interactions: The association for parental GAD was significantly higher in the older age span; the reverse was found for parental depressive and substance use disorders and behavioral inhibition.

Most subthreshold GAD definitions identified cases with more benign risk-factor constellations compared to DSM-IV GAD (see OR1 in Table 5). However, relative to nonGAD cases, individuals with subthreshold GAD frequently still exhibited significant associations with important predictors of GAD (see OR2 in Table 5). Similar findings occurred in both age spans (results available upon request).

Some exceptional findings shall be highlighted: First, regarding parental GAD and early adversity, subthreshold definitions of GAD by and large identified cases not differing from DSM-IV GAD. Of note, not only older but also younger cases identified through most of the subthreshold GAD definitions revealed associations with parental GAD when compared to non-cases. Cases identified by relaxing the associated symptom and clinical significance criteria were not associated with parental GAD specifically but with parental anxiety disorders more broadly. Second, although most of the associations were revealed in both age spans, some significant interactions with age were found, most consistently with regard to parental substance use disorder and reward dependence (higher associations in the older age span for some subthreshold definitions relaxing excessiveness compared to non-cases) as well as behavioral inhibition (higher associations in younger age span for all subthreshold definitions not relaxing excessiveness). Interestingly, when only clinical significance was omitted, younger cases revealed significantly higher reward dependence and older cases lower harm avoidance. Third, some subthreshold GAD definitions with relaxed duration and/or excessiveness revealed more favorable results with regard to novelty seeking in GAD 
compared to non-GAD cases. These counterintuitive findings are due to cases from the older age span.

\section{Discussion}

In light of the forthcoming DSM revision, systematic examinations of diagnostic criteria are needed, particularly in GAD, given controversies on its diagnostic categorization (Beesdo et al., 2010; Mennin et al., 2008) and past criteria changes. In addition to broader appraisals of DSM-IV GAD criteria (Andrews et al., 2010), specific questions arise regarding developmental aspects: Are the current diagnostic criteria for GAD too strict particularly for youth? Is there supporting evidence for different criteria in youth and adults?

Two major findings emerged from the current analyses, conducted in adolescents and young adults, reporting on GAD symptoms between the ages of nine and 34 years. In this sample, relaxing the duration and excessiveness criteria led to the most pronounced increases in GAD prevalence, while relaxing frequency, uncontrollability, and associated symptom criteria had less effect. Importantly, the effects of criteria changes were age-dependent: while a lower duration requirement increased rates more in the older (21-34 years) than the younger (9-20 years) age span, proportionally more younger than older cases were diagnosed when only one and not three physical symptoms were required or when clinical significance was omitted. Second, relative to full-criteria cases, cases identified only with broader GAD definitions in both age spans were frequently of milder severity, less persistent, and less strongly associated with clinical validators. Yet, subthreshold cases generally still differed from non-GAD cases in a range of important, clinically relevant factors.

Before discussing these findings' implications, potential limitations should be considered. First, a person-by-year data file was created, using retrospective age-of-onset and age-ofrecency information; this approach is subject to recall bias. However, this procedure still was able to capture the 'waxing and waning' nature of GAD expressions. Second, some analyses were based on small case numbers, and replication is needed in larger samples. Third, the youngest individuals were aged 14; retrospective information was only considered for ages as low as 9 years to limit recall bias. Our findings, thus, cannot be generalized to younger children. Studies covering broader age ranges are needed. Fourth, this study performed many comparisons, possibly inflating risk of Type I errors. We did not correct for multiple tests because our analysis was exploratory and to prevent penalizing findings in different age spans that were chosen following assumptions in DSM-IV. Moreover, prior research supports independent hypotheses for many of the analyses, making such corrections overly conservative. Fifth, the imperfect retest-reliability of the GAD DSM-IV/M-CIDI diagnosis should be noted. Given that disagreements were shown to be mainly due to the mandatory 6month duration and given that reliability of the GAD stem question was good (Wittchen et al., 1998a), our findings in broader GAD definitions, particularly in the large group with lower time duration, are likely to be based on reliably identified cases. However, uncertainty remains as to whether these new cases are truly new, shorter duration cases or cases of longer duration misspecified due to poor retrospective recall. Sixth, consistent with other studies (Beesdo et al., 2010; Carter et al., 2001; Ruscio et al., 2007), diagnostic exclusion rules for GAD were not applied. Other research evidences little impact of exclusion criteria on GAD prevalence rates (Carter et al., 2001), and even GAD in the absence of comorbidity is characterized by functional impairments and disabilities (Wittchen et al., 2002a). Thus artificial comorbidity e if any e may have had only limited impact on our findings. Seventh, as data to generate diagnoses of OAD were not collected, we were unable to identify how many 
'missed' GAD cases would have been diagnosed using OAD criteria. Eighth, our findings are based on data from a community study which is not bound to selection bias. Other conclusions may emerge from clinical samples.

Given the limitations of this exploratory study, results must be viewed with some caution, particularly pertaining to the novel findings on development that require replication, especially if counterintuitive or inconsistent with prior research in adults (see below).However, many results are comparable with previous studies among adults extending their findings to youth. For example, similar to previous findings (Kessler et al., 2005a; Lee et al., 2009; Ruscio et al., 2007, 2005), our study showed that broader definitions of GAD may identify an additionally, generally milder subgroup of cases that, however, are still characterized by significant burdens in terms of comorbidity, increased help seeking, and persistence of symptomatology. We additionally showed that these cases can be 'validated' as cases based on a broad range of risk variables including familial liability for psychopathology, temperamental/personality profiles, and environmental factors. Overall, results are tentatively suggestive of a severity dimension underlying DSM-IV GAD, with lower thresholds along the dimension generally associated with lesser severity. This generates the difficult question concerning the location on such a continuous 'GAD dimension' where a categorical, diagnostic threshold should be located. While it appears inappropriate to include all subthreshold cases merely due to the finding that these differ in important variables from noncases, it may be suitable in a first step to consider at least the most severe instances of these new cases. However, given the heterogeneity of findings, there appears to be no simple solution. For example, while comorbidity with any substance use disorder was significantly lower in subthreshold compared to full-threshold cases when omitting excessiveness (alone or in combination with other criteria), the same was true for any depressive disorder when lowering duration; comorbidity with anxiety disorders was generally lower only when relaxing multiple criteria. Thus, although a validator-based approach to improve diagnosis and classification has been promoted (Regier et al., 2009), it remains unspecified how to weigh and integrate contrasting findings across validators, to most clearly guide criteria changes. Replication of findings appears to be a major task, particularly if counterintuitive results occur, such as findings of more favorable levels of novelty seeking in some subthreshold GAD definitions compared to non-cases.

Various issues need to be considered when revising diagnostic definitions. Validity and clinical utility are important considerations, as are effects on prevalence. As evidenced in the current study, developmental stage represents another, particularly important variable to consider, as it complicates each of these other considerations. Some validators appear particularly relevant at specific ages. For example, the effects of familial liability for DSM-IV GAD may only manifest at higher ages, whereas temperament may contribute particularly strongly to GAD among youth. Above this, our findings also suggest that changes in distinct symptom criteria produce distinct effects in unique age spans. For example, younger cases identified by many subthreshold definitions also showed associations with parental GAD. Thus, besides the general need to improve the GAD definition according to our current knowledge about this condition, developmental aspects may be more strongly considered in DSM-5. While this may generally apply to both ends of the life spectrum including the elderly, the current study focussed on the first three decades of life, and thus can contribute e in contrast to other studies (Andrews and Hobbs, 2010; Kessler et al., 2005a; Lee et al., 2009; Ruscio et al., 2007, 2005) e some preliminary recommendations for DSM-5 with regard to youth vs. young adult GAD criteria. 
A few specific age-related findings emerged in the current study. Proportionally more younger than older cases were diagnosed when only one and not three symptoms were required or when clinical significance was omitted. These additionally identified young cases were further characterized by particular high levels of behavioral inhibition e an early temperament style that places children at increased risk for anxiety disorders. Thus, findings support the DSMIV rule that requires three symptoms in adults but only one in youth. However, few additional young compared to older cases were identified through this rule because individuals who endorse one or two symptoms commonly also endorse at least three symptoms (Beesdo, 2006; Brown et al., 1995; Carter et al., 2001; Ruscio et al., 2007; Tracey et al., 1997). This suggests that beyond differences in symptom count further age-dependent criteria may be conceivable in DSM-5 to allow for valid and clinically meaningful early identification.

Our study suggests that omitting the clinical significance criterion may be an option to diagnose some e yet also few e more affected young cases. Children and adolescents may not recognize changes in their daily life due to worry letting them deny that their worry impairs daily functioning. Particularly children may also lack cognitive abilities to reflect distress or impairment. Importantly, despite lower comorbidity or persistence these cases are still associated with significant help-seeking comparable to those of full-threshold DSM-IV cases. Additionally omitting clinical significance in youth would also not only identify particularly those young cases (in contrast to adults) that have developed significant GAD-pathology upon their high levels of behavioral inhibition, but also those revealing particularly high reward dependence. Familial aggregation with anxiety e if not GAD e is also enhanced in youth when requiring only one symptom and omitting clinical significance.

Based merely on our prevalence findings, relaxing any other specific DSM-IV criterion per se does not appear promising in order to specifically identify proportionally more youth than adult cases. Very few additional cases are detected by omitting frequency of worry or uncontrollability at any age; many new cases across age result from omitting excessiveness. Particularly surprising in this context is our finding on duration. Contrary to presumptions of Carter et al. (2001) and Hunt et al. (2002) that younger individuals worry for shorter periods of time, we found proportionally higher prevalence rates in the older than the younger age span when requiring only three months duration. The different ages of the studied subjects may serve as a methodological explanation for the different findings, but clinical interpretations are also conceivable. If episodes of worrying tend to become shorter in older age this may express growing efforts to cope with or counteract worrying. Adult cases with shorter duration may also reflect short-lived adaptation processes to life stressors. Another possibility is that the young age group had a higher proportion of individuals with an early onset of GAD, which is a predictor for severity (Campbell et al., 2003; Hoehn-Saric et al.,1993) and therefore for the duration of the disorder. A similar explanation may be that young individuals that meet all other GAD criteria present a clinically severe subgroup that is more likely to be affected by the condition for longer periods of time. The particularly strong associations with behavioral inhibition in the young group indirectly support this view. Overall, more work is needed with regard to age-dependent effects of lowering duration for GAD to 3 months as has been recently proposed for DSM-5 (Andrews et al., 2010). The current work suggests that, in order to allow for valid early identification and diagnosis specifically in youth, additional criteria may require revision. Generally, a considerable amount of new cases in all ages, but particularly in adults, are identified with a lowered duration criterion. Although reliability may be improved compared to a 6-month duration requirement (Wittchen et al., 1998a), the prevalence increase must be carefully considered. As recently shown by Andrews and Hobbs (2010), introduction of other and for GAD novel 
behavioral criteria (such as repeatedly seeking reassurance due to worries, marked avoidance of potentially negative events, or marked time and effort preparing for negative outcomes (Andrews et al., 2010)); may in turn reduce the prevalence to rates comparable of DSM-IV GAD. Unfortunately, the current study cannot provide data with this regard because no behavioral symptoms were assessed in the DSM-IV-CIDI-GAD section. However, our findings referring to the uncontrollability and excessiveness criteria can be indirectly interpreted in support of this proposal. Uncontrollability and excessiveness were found to reveal the highest between-criteria associations, suggesting redundancy. As lack of uncontrollability was rarely and lack of excessiveness was frequently the reason for nondiagnosis in all ages, and given that very few cases report that their worry is excessive but still controllable while the reverse was found more frequently, our findings suggests that uncontrollability could be discarded from the GAD criteria set altogether e a proposal also put forward for DSM-5 (Andrews et al., 2010). This may be particularly relevant for the young where the uncontrollability- excessiveness criteria association was especially pronounced. It remains to be investigated whether similar conclusions would emerge from clinical samples. Given that uncontrollability respectively attempts to control worry play an inherent role in psychological models and treatments of GAD (overview in Behar et al., 2009), evidence must be carefully weighted. It may be conceivable that the way through which uncontrollability is expressed may need to be specified more explicitly in the criteria. Attempts to control or stop worry can reflect a cognitive mechanism, which may be particularly difficult to disentangle for children. Other attempts to decrease worry and anxiety may be described through behavioral symptoms. Although previous criteria for OAD in children included symptoms such as excessive need for reassurance (APA, 1987), these were not considered when subsuming OAD under DSM-IV GAD (APA, 1994). Testing the effects of the proposed behavioral symptoms for DSM-5 GAD has begun for adults (Andrews et al., 2010) but considerable more work is needed, particularly among youth.

With regard to the frequency criterion (worrying more days than not), prior research has provided little data. In the current study, omitting this criterion was rarely the reason for nondiagnosis, thus revealing little impact on prevalence. Worrying can be considered normal e even on a daily basis e if not associated with other symptoms. Interestingly, we found frequency of worry to be associated with other criteria only in the older age span where associations were positive for uncontrollability and associated physical symptoms, but $\mathrm{e}$ surprisingly e negative for excessiveness. Future research needs to replicate these explorative findings and further investigate whether worrying for lesser than $50 \%$ of the time over longer time periods (e.g. 3 months) e combined with other symptoms e may already reflect clinical relevance, particularly among children.

To conclude, the current study suggests that age-dependent criteria for GAD should be more systematically explored for DSM-5 to allow for valid identification of clinically relevant cases, particularly among youth. 


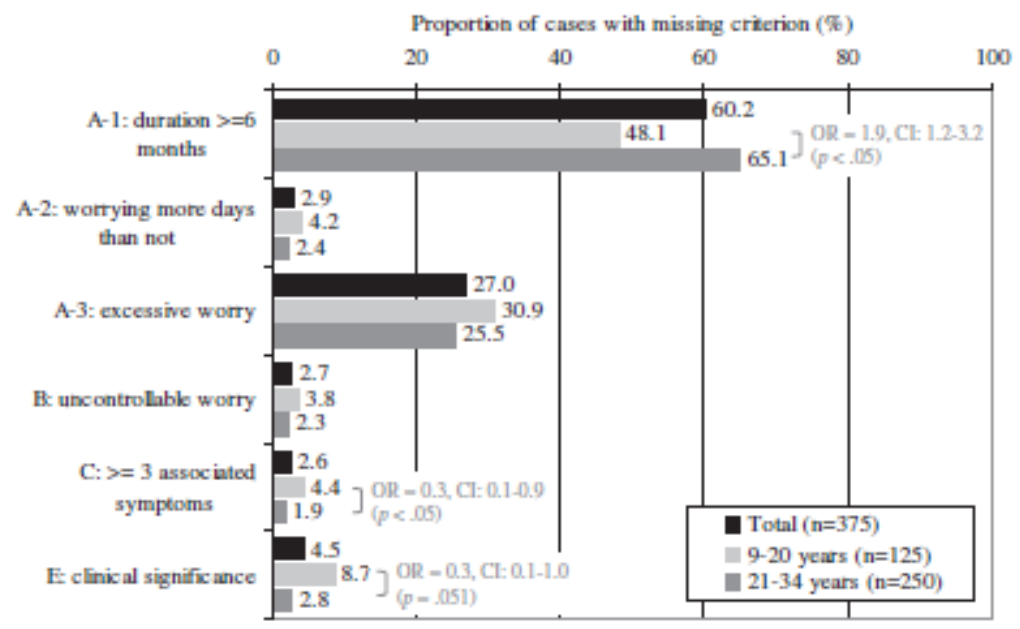

Fig 1. Missing criterion among cases that fell below the threshold of DSM-IV GAD disgnosis by onecrizerian. Cases fom a persan-by-year file (n umweighted; $\chi$ weighted) OR: Odd Rabo with $95 x$ Confidence intenal from logistic regression, adjusted for sex, reference group: individuals from younger age spar.

Table 1

Prevalence of CAD as a function of varying threshold leveds and age.

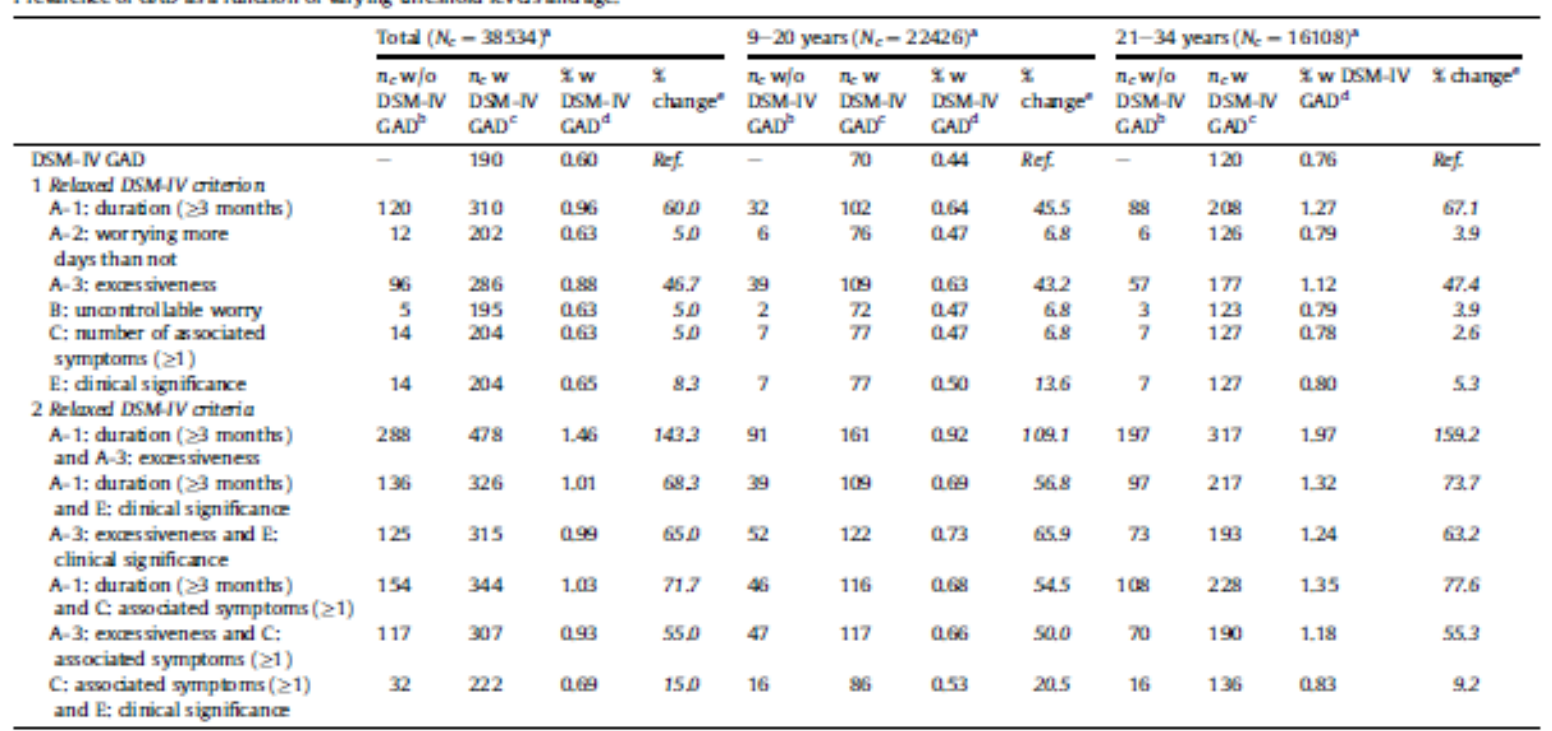

A Cases from a person-by-year file (numbers umweighted)

"Unweighted number of cases with subthreshold CAD alane (without DSM-IV CAD cases)

c Unweighted number of cases with subthreshold or DSM-NV CAD.

a Prevalence of cases with subthresho ld or DSM-IV GAD in perent, percentages weighted [note that the same urnweighted n may currespond with different weighted percentages because the respective cases have different individual sampling weights].

" Incr aase of overall prevalence in percent when compared to DSM-IV CAD (Reference). 
Table 2

Associations between CAD criteria among cases with at least 3 months of worrying

\begin{tabular}{|c|c|c|c|c|c|}
\hline \multirow[t]{2}{*}{ DSM CAD criteria } & \multirow{2}{*}{$\begin{array}{l}\text { A-2: worrying } \\
\text { more days } \\
\text { than not } \\
\mathrm{OK}^{\prime}\end{array}$} & \multirow{2}{*}{$\begin{array}{l}\text { A-3: } \\
\text { exces siveness } \\
\mathrm{OR}^{\mathrm{A}}\end{array}$} & \multirow{2}{*}{$\begin{array}{l}\text { B: } \\
\text { uncontrol lable } \\
\text { worry } \\
\mathrm{OR}^{\wedge}\end{array}$} & \multirow{2}{*}{$\begin{array}{l}\mathrm{C}: \geq 3 \\
\text { associated } \\
\text { symptoms } \\
\mathrm{OK}^{4}\end{array}$} & \multirow{2}{*}{$\begin{array}{l}\text { E: dinical } \\
\text { significance } \\
\\
\mathrm{OR}^{\mathrm{A}}\end{array}$} \\
\hline & & & & & \\
\hline A-1: 6 -month duration & 15 & $15^{*}$ & $1.7^{*}$ & $27^{*}$ & a9 \\
\hline A-2: worrying more dyys than not & - & a.6 & $29^{\circ}$ & $25^{*}$ & 1.0 \\
\hline A-3: excressiveness & & - & $53^{\circ}$ & $18^{*}$ & $41^{*}$ \\
\hline B: uncontrol lable warry & & & - & $36^{*}$ & $33^{\circ}$ \\
\hline A-1: 6-month duration & 13 & $1.8^{*}$ & $20^{*}$ & $29^{*}$ & a.7 \\
\hline A-2: wor rying more dyys than not & - & 1.2 & 1.4 & 0.7 & 1.6 \\
\hline A-3: excessiveness & & - & $122^{*}$ & $28^{*}$ & $36^{\circ}$ \\
\hline B: uncontrol lable warry & & & - & $3.1^{*}$ & $92^{*}$ \\
\hline$C: \geq 3$ associated symptoms & & & & - & 1.8 \\
\hline \multicolumn{6}{|l|}{$21-34$ years } \\
\hline A-1: 6 -month duration & 1.7 & 1.4 & 1.5 & $27^{*}$ & 1.0 \\
\hline
\end{tabular}

" Odd Ratio from logistic regression, adjus ted for age and sex in total group, adjusted for sex in age spars; " $\mathrm{p}<.05$

Table 3

Associations between subthres hold CAD and other mental disorders

\begin{tabular}{|c|c|c|c|c|c|c|c|c|}
\hline \multirow[t]{2}{*}{ Subtreshold CAD } & \multicolumn{2}{|c|}{$\begin{array}{l}\text { Arny comarbid } \\
\text { disorder }\end{array}$} & \multicolumn{2}{|c|}{$\begin{array}{l}\text { Any comorbid } \\
\text { anxiety diso rder }\end{array}$} & \multicolumn{2}{|c|}{$\begin{array}{l}\text { Arny comortid } \\
\text { depressive } \\
\text { disorder }\end{array}$} & \multicolumn{2}{|c|}{$\begin{array}{l}\text { Any comarbid } \\
\text { sukstance use } \\
\text { disorder }\end{array}$} \\
\hline & $\overline{\mathrm{OR} 1^{*}}$ & $\mathrm{OR} 2^{2}$ & $\overline{\mathrm{OR} 1^{*}}$ & OR: & $\overline{\mathrm{OR} 1^{*}}$ & $\mathrm{OR}^{\mathrm{B}}$ & $\overline{\mathrm{OR} 1^{*}}$ & ORZ \\
\hline \multicolumn{9}{|l|}{1 DSM-N Giterian relaxed } \\
\hline A-1: duration ( $\geq 3$ months) & a.* & $88^{*}$ & 0.3 & $73^{*}$ & a.t* & $64^{\circ}$ & 12 & $60^{*}$ \\
\hline A-3: excessiveness & $04^{*}$ & $113^{*}$ & 10 & $99^{*}$ & a9 & $95^{\circ}$ & $02^{*}$ & 1.1 \\
\hline C. number of associated symptoms $(\geq 1)$ & e & c & 10 & $113^{*}$ & a9 & $127^{*}$ & 2.1 & $122^{*}$ \\
\hline E: di rical significance & d & d & d & & d & & d & \\
\hline \multicolumn{9}{|l|}{2 DSM- $N$ aiteria relaned } \\
\hline A-1: duration ( $\geq 3$ months) and A-3; excessiveness & $02^{*}$ & $63^{\circ}$ & $0.5^{*}$ & $56^{*}$ & $a G^{*}$ & $66^{\circ}$ & $0.5^{*}$ & $28^{*}$ \\
\hline A-1: duration ( $\geq 3$ months ) and E: clinical significance & $a 2^{*}$ & $6.1^{*}$ & $06^{*}$ & $53^{*}$ & as. & $52^{*}$ & 10 & $52^{*}$ \\
\hline A-3: excessiveness and E: clinical si gri ficance & $a 2^{*}$ & $6 \% *$ & $0.5^{*}$ & $57^{*}$ & a.7 & $68^{*}$ & $0.3^{*}$ & 15 \\
\hline A-1: duration ( $\geq 3$ months ) and $C:$ associated symptoms ( $\geq 1)$ & a.* & $91^{*}$ & 03 & $72^{*}$ & $a 6^{*}$ & $7.1^{*}$ & 1.1 & $58^{*}$ \\
\hline A-3: excessiveress and C: ssociated symptoms ( $\geq 1)$ & $04^{*}$ & $113^{*}$ & 09 & $93^{*}$ & a9 & $9 g^{*}$ & $0 A^{*}$ & 13 \\
\hline C assodixed symptoms $(\geq 1)$ and E: dinical significance & $\mathrm{a}_{1}$. & 20 & $0.2^{*}$ & 13 & $0.3^{*}$ & $3.1^{*}$ & 06 & $30^{*}$ \\
\hline
\end{tabular}

Table 4

Validators of DSM-IV CAD in the total sample and by age

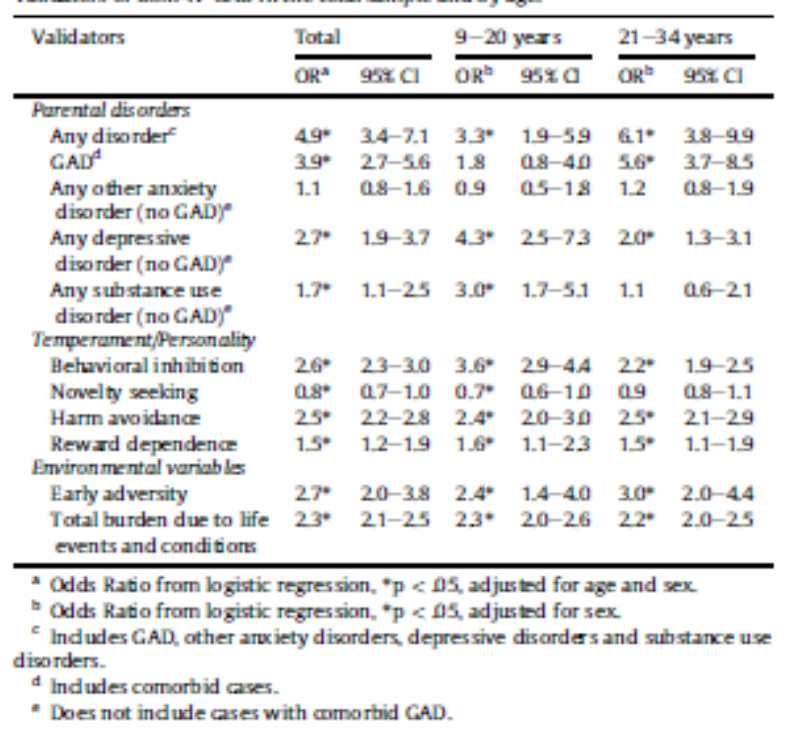




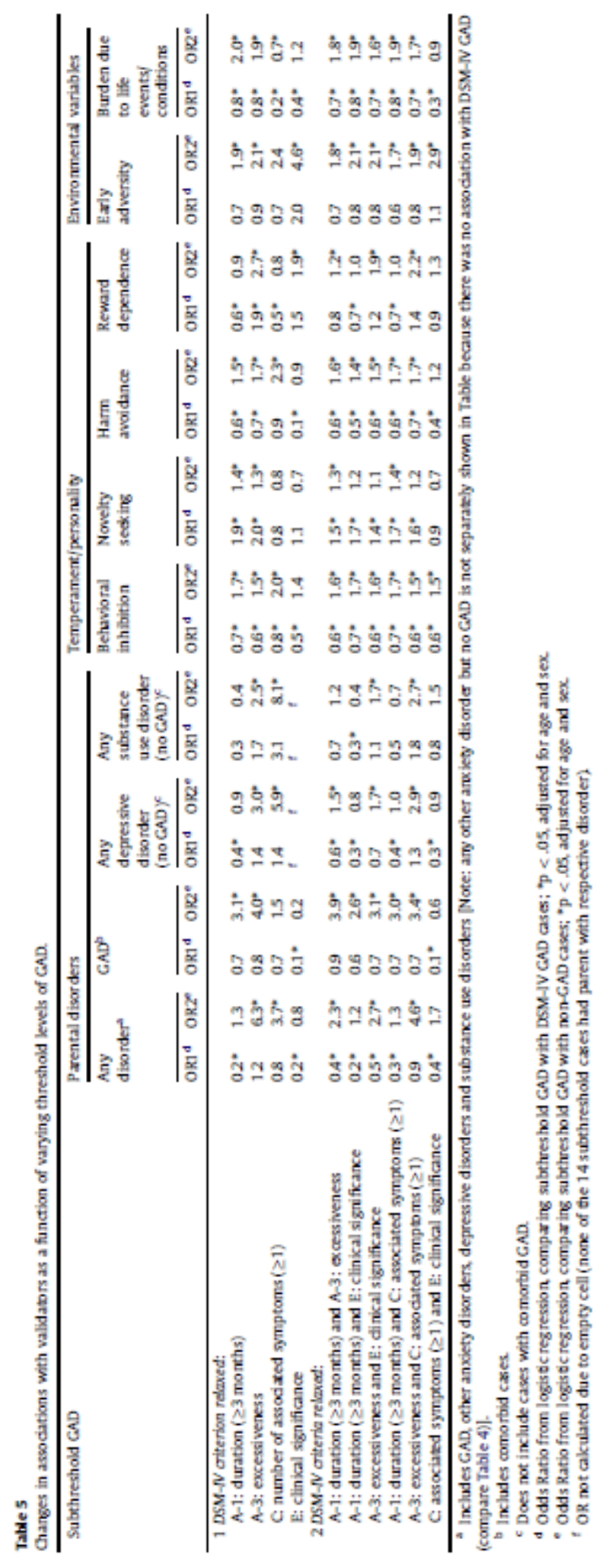

\section{Role of funding source}

This work is part of the Early Developmental Stages of Psychopathology (EDSP) Study which was funded by the German Federal Ministry of Education and Research (BMBF) project no. 01EB9405/6, 01EB 9901/6, EB01016200, 01EB0140, and 01EB0440. Part of the field work and analyses were also additionally supported by grants of the Deutsche 
Forschungsgemeinschaft (DFG) LA1148/1-1, WI2246/1-1, WI 709/7-1, and WI 709/8-1. The funding sources had no further role in study design, in the collection, analysis and interpretation of data, in the writing of the report, and in the decision to submit the paper for publication.

\section{Contributors}

Dr. Roselind Lieb and Dr. Hans-Ulrich Wittchen are the principal investigators of the EDSP and designed the study. Dr. Katja Beesdo-Baum and Dipl.-Psych. Susanne Winkel managed the literature searches, performed the statistical analyses, and wrote the first draft of the manuscript. Dr. Michael Höfler supported the statistical analyses. Dr. Daniel S. Pine, Dr. Jürgen Hoyer, Dr Roselind Lieb and Dr. Hans-Ulrich Wittchen provided critical feedback and supported data interpretation. All authors contributed to and approved the final manuscript. Dr. Beesdo-Baum was the primary author.

\section{Conflict of interest}

Dr. Beesdo-Baum has received speaking honoraria from Pfizer and Eli Lilly. Dr. Wittchen has received speaking honoraria from Eli Lilly and Company, Pfizer and Norvartis and serves on advisory boards for Pfizer, Servier and Schering-Plough. Dipl.-Psych. Winkel, Dr. Höfler, Dr. Hoyer, Dr. Pine and Dr. Lieb have nothing to declare.

\section{Acknowledgments}

Principal investigators of the EDSP are Dr. Hans-Ulrich Wittchen and Dr. Roselind Lieb. Core staff members of the EDSP group are: Dr. Kirsten von Sydow, Dr. Gabriele Lachner, Dr. Axel Perkonigg, Dr. Peter Schuster, Dr. Michael Höfler, Dipl.-Psych. Holger Sonntag, Dipl.-Psych. Tanja Brückl, Dipl.-Psych. Elzbieta Garczynski, Dr. Barbara Isensee, Dipl.Psych. AgnesNocon,Dr. ChrisNelson,Dipl.-Inf. Hildegard Pfister,Dr.VictoriaReed,Dipl.-Soz. BarbaraSpiegel,Dr.AndreaSchreier, Dr.UrsulaWunderlich,Dr. Petra Zimmermann, Dr.Katja Beesdo-Baum, Dr. Antje Bittner, Dr. Silke Behrendt and Dr. Susanne Knappe. Scientific advisors are Dr. Jules Angst (Zurich), Dr. Jürgen Margraf (Basel), Dr. Günther Esser (Potsdam), Dr. Kathleen Merikangas (NIMH, Bethesda), Dr. Ron Kessler (Harvard, Boston) and Dr. Jim van Os (Maastricht).

\section{References}

- Andrews G, Hobbs M. The effect of the draft DSM-5 criteria for GAD on prevalence and severity. Australian and New Zealand Journal of Psychiatry 2010;44:784-90.

- Andrews G, Hobbs MJ, Borkovec TD, Beesdo K, Craske MG, Heimberg RG, et al. Generalized worry disorder: a review of DSM-IV generalized anxiety disorder and options for DSM-V. Depression and Anxiety 2010;27:134-47.

- APA. Diagnostic and statistical manual of mental disorders. 3rd ed. Washington, D.C.: American Psychiatric Press; 1980.

- APA. Diagnostic and statistical manual of mental disorders. revised 3rd ed. Washington, D.C.: American Psychiatric Press; 1987.

- APA. Diagnostic and statistical manual of mental disorders. 4th ed. Washington, D.C.: American Psychiatric Press; 1994.

- Beesdo K. Wie entstehen Generalisierte Ängste? Eine prospektiv-longitudinale, klinisch-epidemiologische Studie bei Jugendlichen und jungen Erwachsenen [The development of Generalized Anxiety? A prospective-longitudinal, 
clinicalepidemiologic study among adolescents and young adults]. Dresden: TUD Press; 2006.

- Beesdo K, Knappe S, Pine DS. Anxiety and anxiety disorders in children and adolescents: developmental issues and implications for DSM-V. Psychiatric Clinics of North America 2009;32:483-524.

- Beesdo K, Pine DS, Lieb R, Wittchen HU. Incidence and risk patterns of anxiety and depressive disorders and categorization of generalized anxiety disorder. Archives of General Psychiatry 2010;67:47-57.

- Behar E, Dobrow DiMarco I, Hekler EB, Mohlman J, Staples AM. Current theoretical models of generalized anxiety disorder (GAD): conceptual review and treatment implications. Journal of Anxiety Disorders 2009;23:1011-23.

- Bienvenu OJ, Nestadt G, Eaton WW. Characterizing generalized anxiety: temporal and symptomatic thresholds. Journal of Nervous and Mental Disease 1998;186:51-6.

- Bittner A, Egger HL, Erkanli A, Costello EJ, Foley DL, Angold A.What do childhood anxiety disorders predict? Journal of Child Psychology and Psychiatry 2007;48:117483.

- Brown TA, Barlow DH, Liebowitz MR. The empirical basis of generalized anxiety disorder. American Journal of Psychiatry 1994;151:1272-80.

- Brown TA, Di Nardo PA, Lehman CL, Campbell LA. Reliability of DSM-IV anxiety and mood disorders: implications for the classification of emotional disorders. Journal of Abnormal Psychology 2001;110:49-58.

- Brown TA, Marten PA, Barlow DH. Discriminant validity of the symptoms constituting the DSM-III-R and DSM-IV associated symptom criterion of generalized anxiety disorder. Journal of Anxiety Disorders 1995;9:317-28.

- Campbell LA, Brown NA, Grisham JR. The relevance of age of onset to the psychopathology of generalized anxiety disorder. Behavior Therapy 2003;34:31-48.

- Carter RM, Wittchen H-U, Pfister H, Kessler RC. One-year prevalence of subthreshold and threshold DSM-IV generalized anxiety disorder in a nationally representative sample. Depression and Anxiety 2001;13:78-88.

- Cloninger C. Neurogenetic adaptive mechanisms in alcoholism. Science 1987a;236:410-6.

- Cloninger C. A systematic method for clinical description and classification of personality variants. Archives of General Psychiatry 1987b;44:576-88.

- Cohen P, Cohen J, Brook J. An epidemiological study of disorders in late childhood and adolescence e II. Persistence of disorders. Journal of Child Psychology and Psychiatry and Allied Disciplines 1993;34:869-77.

- Friis RH, Wittchen H-U, Pfister H, Lieb R. Life events and changes in the course of depression in young adults. European Psychiatry 2002;17:241-53.

- Goldberg D. Towards DSM-V: the relationship between generalized anxiety disorder and major depressive episode. Psychological Medicine 2008;38:1671-5.

- Hettema JM. The nosological relationship between generalized anxiety disorder and major depression. Depression and Anxiety 2008;25:300-16.

- Hoehn-Saric R, Hazlett RL, McLeod DR. Generalized anxiety disorder with early and late onset of anxiety symptoms. Comprehensive Psychiatry 1993;34:291-8.

- Hoyer J, Becker E, Margraf J. Generalized anxiety disorder and clinical worry episodes in young women. Psychological Medicine 2002;32:1227-37.

- Hoyer J, Becker ES, Roth WT. Characteristics of worry in GAD patients, social phobics, and controls. Depression and Anxiety 2001;13:89-96. 
- Hunt C, Issakidis C, Andrews G. DSM-IV generalized anxiety disorder in the Australian National Survey of mental health and well-being. Psychological Medicine 2002;32:649-59.

- Kendall PC, Warman MJ. Anxiety disorders in youth: diagnostic consistency across DSM-III-R and DSM-IV. Journal of Anxiety Disorders 1996;10:453-63.

- Kessler RC, Brandenburg N, Lane M, Roy-Byrne P, Stang PE, Stein DJ, Wittchen HU. Rethinking the duration requirement for generalized anxiety disorder: evidence from the National Comorbidity Survey Replication. Psychological Medicine 2005a;35:1073-82.

- Kessler RC, Chiu WT, Demler O, Walters EE. Prevalence, severity, and comorbidity of 12-month DSM-IV disorders in the National Comorbidity Survey Replication. Archives of General Psychiatry 2005b;62:617-27.

- Kessler RC, Gruber M, Hettema JM, Hwang I, Sampson N, Yonkers KA. Co-morbid major depression and generalized anxiety disorders in the National Comorbidity Survey follow-up. Psychological Medicine 2008;38:365-74.

- Lee S, Tsang A, Ruscio AM, Haro JM, Stein DJ, Alonso J, et al. Implications of modifying the duration requirement of generalized anxiety disorder in developed and developing countries. Psychological Medicine 2009;39:1163-76.

- Lieb R, Isensee B, Höfler M, Pfister H, Wittchen H-U. Parental major depression and the risk of depressive and other mental disorders in offspring: a prospectivelongitudinal community study. Archives of General Psychiatry 2002;59:365-74.

- Lieb R, Isensee B, von Sydow K, Wittchen H-U. The early developmental stages of psychopathology study (EDSP): a methodological update. European Addiction Research 2000;6:170-82.

- Maier-Diewald W, Wittchen H-U, Hecht H, Werner-Eilert K. Die Münchner Ereignisliste (MEL) - Anwendungsmanual. München: Max-Planck-Institut für Psychiatrie, Klinische Psychologie und Epidemiologie (Eigendruck); 1983.

- Marten PA, Brown TA, Barlow DH, Borkovec TD, Shear MK, Lydiard RB. Evaluation of the ratings comprising the associated symptom criterion of DSM-III-R generalized anxiety disorder. Journal of Nervous and Mental Disease 1993;181:67682.

- Mennin DS, Heimberg RG, Fresco DM, Ritter MR. Is generalized anxiety disorder an anxiety or mood disorder? Considering multiple factors as we ponder the fate of GAD. Depression and Anxiety 2008;25:289-99.

- Merikangas KR, Mehta RL, Molnar BE, Walters EE, Swendsen JD, Aguilar-Gaziola $\mathrm{S}$, et al. Comorbidity of substance use disorders with mood and anxiety disorders: results of the international consortium in psychiatric epidemiology. Addictive Behaviors 1998;23:893-907.

- Moffitt TE, Caspi A, Harrington H, Milne BJ, Melchior M, Goldberg D, Poulton R. Generalized anxiety disorder and depression: childhood risk factors in a birth cohort followed to age 32. Psychological Medicine 2007;37:441-52.

- Pine DS, Cohen P, Gurley D, Brook J, Ma Y. The risk for early-adulthood anxiety and depressive disorders in adolescents with anxiety and depressive disorders. Archives of General Psychiatry 1998;55:56-64.

- Reed V, Gander F, Pfister H, Steiger A, Sonntag H, Trenkwalder C, et al. To what degree the Composite International Diagnostic Interview (CIDI) correctly identify DSM-IV disorders? Testing validity issues in a clinical sample. International Journal of Methods in Psychiatric Research 1998;7:142-55.

- Regier DA, Narrow WE, Kuhl EA, Kupfer DJ. The conceptual development of DSMV. American Journal of Psychiatry 2009;166:645-50. 
- Reznick JS, Hegeman IM, Kaufman ER, Woods SW, Jacobs M. Retrospective and concurrent self-report of behavioral inhibition and their relation to adult mental health. Development and Psychopathology 1992;4:301-21.

- Rohrbacher H, Hoyer J, Beesdo K, Höfler M, Bittner A, Lieb R, Wittchen H-U. Psychometric properties of the retrospective self report of inhibition (RSRI) in a representative German sample. International Journal of Methods in Psychiatric Research 2008; 17:80-8.

- Royall RM. Model robust confidence intervals using maximum likelihood estimators. International Statistical Review 1986;54:221-6.

- Ruscio AM. Delimiting the boundaries of generalized anxiety disorder: differentiating high worries with and without GAD. Journal of Anxiety Disorders 2002;16:377-400.

- Ruscio AM. Integrating structural and epidemiological research to inform the classification of psychopathology. International Journal of Methods in Psychiatric Research; 2009; doi:10.1002/mpr.295.

- Ruscio AM, Borkovec TD, Ruscio J. A taxometric investigation of the latent structure of worry. Journal of Abnormal Psychology 2001;110:413-22.

- Ruscio AM, Chiu WT, Roy-Byrne P, Stang PE, Stein DJ, Wittchen H-U, Kessler RC. Broadening the definition of generalized anxiety disorder: effects on prevalence and associations with other disorders in the National Comorbidity Survey Replication. Journal of Anxiety Disorders 2007;21:662-76.

- Ruscio AM, Lane M, Roy-Byrne P, Stang PE, Stein DJ, Wittchen H-U, Kessler RC. Should excessive worry be required for a diagnosis of generalized anxiety disorder? Results from the US National Comorbidity Survey Replication. Psychological Medicine 2005;35:1761-72.

- Stata Corp. Stata statistical software: release 11.0. College Station, TX: Stata Corporation; 2009.

- Tracey SA, Chorpita BF, Douban J, Barlow DH. Empirical evaluation of DSM-IV generalized anxiety disorder criteria in children and adolescents. Journal of Clinical Child Psychology 1997;26:404-14.

- Velez CN, Johnson J, Cohen P. A longitudinal analysis of selected risk factors for childhood psychopathology. Journal of the American Academy of Child and Adolescent Psychiatry 1989;28:861-4.

- Weyers P, Krebs J, Janke W. Reliability and construct validity of the German version of Cloninger's tridimensional personality questionnaire. Personality and Individual Differences 1995;9:853-61.

- Wittchen H-U, Beesdo K, Kessler RC. The impact of generalized anxiety disorder. In: Nutt DJ, Rickels K, Stein DJ, editors. Generalized anxiety disorder: symptomatology, pathogenesis and management. London: Martin Dunitz; 2002a. p. 11-26.

- Wittchen H-U, Kessler RC, Beesdo K, Krause P, Höfler M, Hoyer J. Generalized anxiety and depression in primary care: prevalence, recognition and management. Journal of Clinical Psychiatry 2002b;63:24-34.

- Wittchen H-U, Kessler RC, Zhao S, Abelson J. Reliability and clinical validity of UMCIDI DSM-III-R generalized anxiety disorder. Journal of Psychiatric Research 1995;29:95-110.

- Wittchen H-U, Lachner G, Wunderlich U, Pfister H. Test-retest reliability of the computerized DSM-IV version of the Munich-Composite International Diagnostic Interview (M-CIDI). Social Psychiatry and Psychiatric Epidemiology 1998a;33:56878. 
- Wittchen H-U, Lieb R, Pfister H, Schuster P. The waxing and waning of mental disorders: evaluating the stability of syndromes of mental disorders in the population. Comprehensive Psychiatry 2000;41:122-32.

- Wittchen H-U, Lieb R, Schuster P, Oldehinkel AJ. When is onset? Investigations into early developmental stages of anxiety and depressive disorders. In: Rapoport JL, editor. Childhood onset of "adult" psychopathology, clinical and research advances. Washington: American Psychiatric Press; 1999. p. 259-302.

- Wittchen H-U, Nelson CB, Lachner G. Prevalence of mental disorders and psychosocial impairments in adolescents and young adults. Psychological Medicine 1998b;28:109-26.

- Wittchen H-U, Perkonigg A, Lachner G, Nelson CB. Early developmental stages of psychopathology study (EDSP) e objectives and design. European Addiction Research 1998c;4:18-27.

- Wittchen H-U, Pfister H. DIA-X-Interviews: Manual für Screening-Verfahren und Interview; Interviewheft Längsschnittuntersuchung (DIA-X-Lifetime);

Ergänzungsheft (DIA-X-Lifetime); Interviewheft Querschnittuntersuchung (DIA-X-12 Monate); Ergänzungsheft (DIA-X-12Monate); PC-Programm zur Durchführung des Interviews (Längs- und Querschnittuntersuchung); Auswertungsprogramm. Frankfurt: Swets \& Zeitlinger; 1997. 